\section{Wipe away your infection control fears}

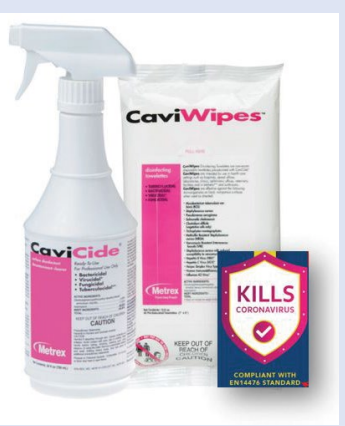

CaviWipes from Kerr are all-inone, ready-to use disinfectant wipes pre-saturated with CaviCide solution for the effective removal of dirt as well as quick and broad-spectrum disinfection.

Available in a flat pack or cannister for convenience, CaviWipes have a low alcohol content (17\%), preventing

bioburden from becoming insoluble and stubbornly adherent to surfaces, for a more effective clean and disinfection. They do not contain aldehydes, phenols, bleach or other toxic chemicals.

CaviCide is effective against human coronavirus and with these extra-large and thick wipes, fungicidal, bactericidal, virucidal and tuberculocidal disinfection is achieved in just three minutes.

In addition, the non-woven fabric allows the tissue to remain intact, without bunching up or drying out during use.

CaviWipes are award winners: The Dental Advisor 2017 Award Winner -Surface Disinfectant and The Dental Advisor 2018 - Preferred Product - Surface Disinfectant.

For further information, visit www.kerrdental.com/en-uk or contact your local rep.

\section{Drive better outcomes}

With quality diagnostic scans come quality restorations. So why not ensure that you are able to drive better outcomes than ever before with the CS 3700 intraoral scanner from Carestream Dental?

Innovative shade-match technology ensures that achieving outstanding aesthetics is incredibly simple, while the hybrid scan ability allows you to merge a partial impression with a digital in vivo scan into a single file. Plus, the scanner has increased scan space real estate, providing a clearer view of the live scan for more accurate diagnoses.

For more information, contact Carestream Dental on 0800169 9692 or visit www.carestreamdental.co.uk.

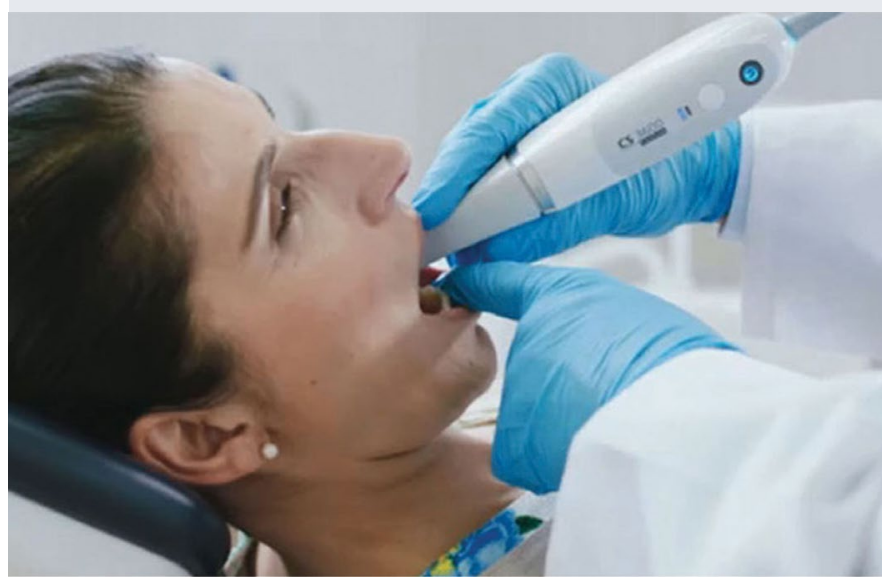

\section{Aesthetic even for bleached dentition}

With more and more people undergoing cosmetic treatments such as tooth whitening, it can be difficult to find a composite in the right shade.

With Filtek Universal Restorative from 3M, you can restore even the whitest teeth with confidence using the Extra White option that covers all Vita bleach shades.

Furthermore, Filtek Universal Restorative features 3M's innovative NaturalMatch technology with proprietary lowstress monomers, which, when combined with the inbuilt TRUE nanotechnology, helps to reduce shrinkage and stress [3M internal data].

For more information, call 08705360036 or visit 3m.co.uk/ filtek-universal. $3 \mathrm{M}$ representatives remain contactable by phone or via video conferencing.

$3 \mathrm{M}$ and Filtek are trademarks of the 3M Company.

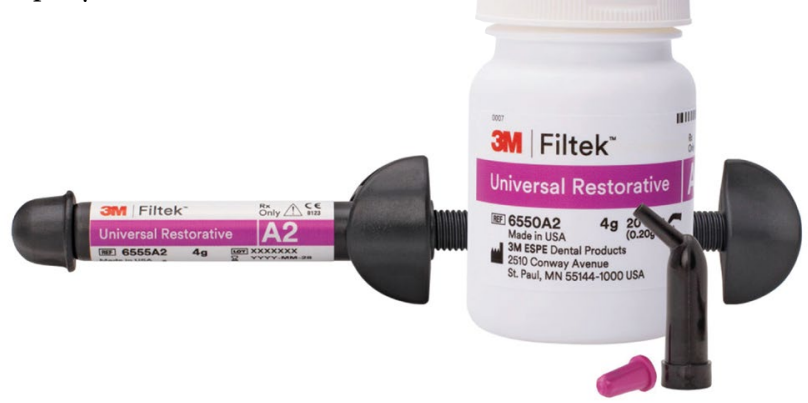

\section{Minimise the production of contaminated aerosols}

To support dental professionals, W\&H dental turbines offer features to minimise the production of contaminated aerosols in practice.

These handpieces feature a patented hygiene head with an antiretraction system that is designed to limit the number of aerosols being sucked back into the turbine as the rotor slows to a stop.

Dental turbines from W\&H have also been optimised with an anti-retraction valve within the turbine itself, which prevents the backflow of potentially contaminated water via the turbine's waterlines to the central water supply system.

This facilitates the highest infection control standards for ultimate peace-of-mind among both staff and patients.

Discover the full range of $\mathrm{W} \& \mathrm{H}$ handpieces via the website today.

To find out more visit www.wh.com/en_uk, call 01727874990 or email office.uk@wh.com.

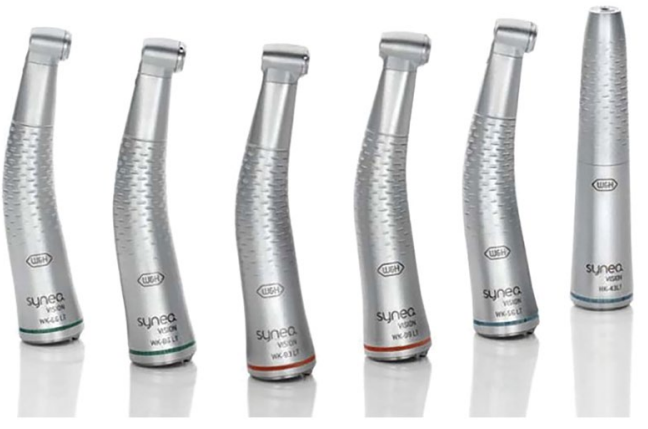

